\title{
Necrotic arm lesions in an intravenous drug user
}

\author{
Amesh Adalja $\cdot$ Anjum S. Kaka
}

Received: 12 January 2010/Accepted: 10 February 2010/Published online: 14 April 2010

(C) SIMI 2010

\section{Case report}

A 62-year-old woman with a history of long-term intravenous drug use and chronic Hepatitis $C$ virus infection was admitted to another hospital unresponsive with profound hypotension. Her symptoms were attributed to a drug overdose leading to a depressed mental status, and possibly septic shock with aspiration pneumonia. She was placed on mechanical ventilation, and treated with vasopressors, high-dose corticosteroids and several broad-spectrum antibiotics. Blood and urine cultures were negative. When she failed to improve after 1 week she was transferred to the intensive care unit at our institution. On transfer, she was unresponsive, intubated, on mechanical ventilation, and hypotensive on vasopressors. We also noted that she had multiple ulcerated skin lesions on both upper extremities (Fig. 1). The lesions involved the dorsum of both hands and wrists, medial aspects of both arms, and the left antecubital fossa. The edges of the lesions were necrotic and the base was macerated.

The WBC count was $22,500 \mathrm{~mm}^{-3}$ with $85 \%$ neutrophils and $7 \%$ band forms. The creatinine was $3 \mathrm{mg} / \mathrm{dL}$. An acute renal failure was attributed to acute tubular necrosis. HIV testing was negative. The CT scans of the chest, abdomen, pelvis, and MRI of the head were unrevealing,

\section{A. Adalja $(\bowtie)$. A. S. Kaka}

Division of Infectious Diseases, Department of Medicine, University of Pittsburgh Medical Center, 3550 Terrace Street, Pittsburgh, PA 15261, USA

e-mail: AmeshAA@aol.com

A. Adalja

Center for Biosecurity of University of Pittsburgh

Medical Center, Baltimore, MD, USA and a lumbar puncture was unremarkable. A skin biopsy of the leading edge of the ulcer was performed.

\section{Diagnosis: primary cutaneous mucormycosis}

Cutaneous mucormycosis was diagnosed via histopathology results and cultures. The skin biopsy showed necrotic tissue with broad, ribbon-like, pauci-septate fungal hyphae within the dermis consistent with cutaneous mucormycosis (Fig. 2). There was tissue invasion as evidenced by the presence of hyphal elements within the blood vessels and surrounding inflammatory infiltrate (Fig. 3). Cultures of the skin biopsy were negative for bacterial pathogens, but fungal cultures in Sabouraud's agar were positive for Rhizomucor.

The patient underwent extensive debridement of both upper extremities. She was initially treated with liposomal amphotericin B at a dose of $5 \mathrm{mg} / \mathrm{kg} /$ day. The steroids were weaned, and the serum glucose was strictly controlled. Despite repeated debridements, the skin lesions continued to progress, ultimately culminating in bilateral upper extremity amputations. In lieu of progression of the disease on amphotericin $\mathrm{B}$, the antifungal regimen was switched to posaconazole as salvage therapy for refractory cutaneous mucormycosis. Unfortunately, the patient continued to remain ventilator and vasopressor-dependent, and her family withdrew care. A post-mortem examination was refused.

Rhizomucor is one of the etiologic agents of mucormycosis, and causes systemic infections mostly in immunecompromised hosts [1]. Primary cutaneous mucormycosis has been described in both immune-compromised and immune-competent persons who have devitalized tissue after major trauma or burn wounds, minor skin trauma such 


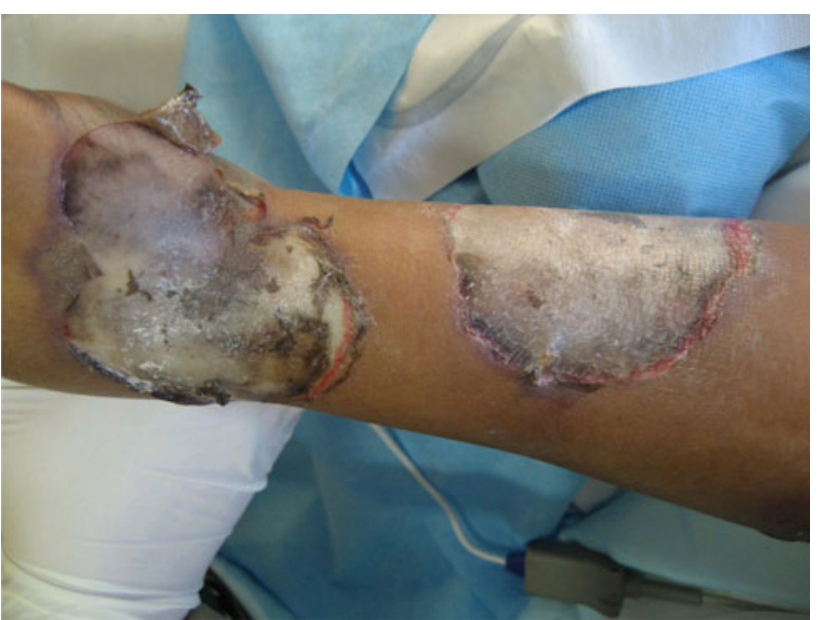

Fig. 1 Photograph of the lesion

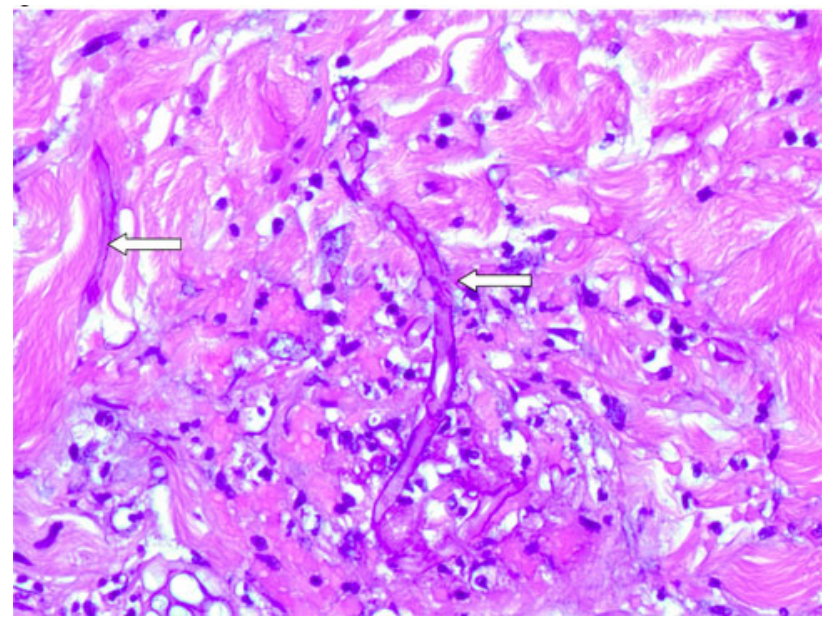

Fig. 2 Photomicrograph 1 of the biopsy of the leading edge of the skin ulcer stained using the Periodic acid Schiff (PAS) stain $(\times 60)$ showing PAS positive, irregular, ribbon-shaped, pauci-septate hyphal elements (white arrows)

as from placement of intravenous catheters, or those who receive topical dressings contaminated with fungal spores $[2,3]$. Biopsy is required for definitive diagnosis.

This patient had cutaneous fungal infection restricted to her arms, around the sites of superficial veins. She did not have any peripheral IVs or dressings in the involved areas. The spores of mucormycosis are ubiquitous, and are likely present in illicit drugs (illicit drugs have also been hypothesized to be the source of fungal spores causing

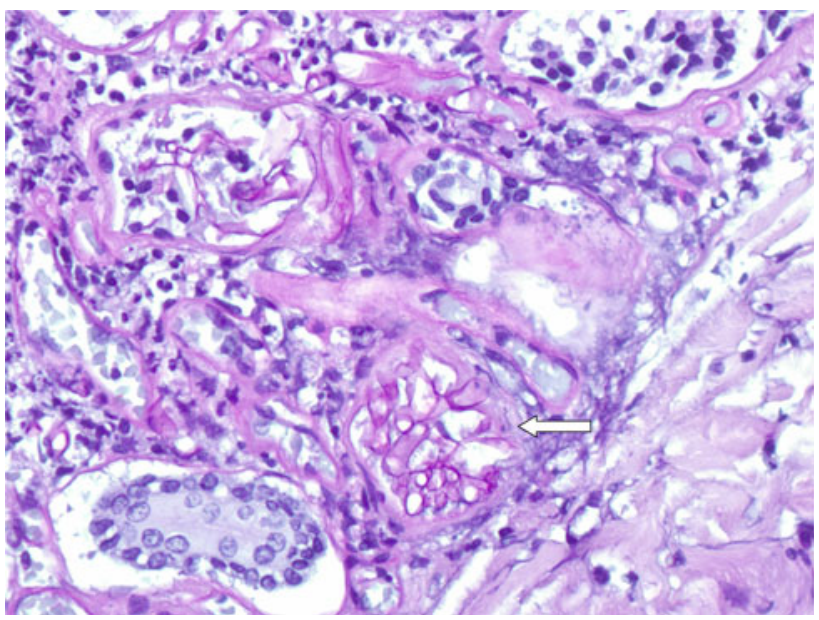

Fig. 3 Photomicrograph 2 of the biopsy of the leading edge of the skin ulcer stained using the Periodic acid Schiff (PAS) stain $(\times 60)$ showing PAS positive hyphal elements clustered within a blood vessel (white arrow)

isolated cerebral mucormycosis in immunocompetent injection drug users) [4]. Hence, we propose that during drug injection our patient inoculated fungal spores into the superficial tissues; the combination of local tissue trauma, systemic hypotension, broad-spectrum antibiotics, highdose steroid use and poor glucose control in the ICU likely contributed to an ideal environment for the inoculated spores to germinate, and resulted in primary invasive cutaneous mucormycosis at multiple injection sites resulting in septic shock due to disseminated fungal infection.

Conflict of interest None.

\section{References}

1. Sugar M (2005) Agents of mucormycosis and related species. In: Mandell GL, Bennett JE, Dolin R (eds) Principles and practice of infectious diseases. Churchill Livingston, Philadelphia

2. Dennis JE, Rhodes KH, Cooney DR, Roberts GD (1980) Nosocomical Rhizopus infection (zygomycosis) in children. J Pediatr 96:824-828

3. Gartenberg G, Bottone EJ, Keusch GT, Weitzman I (1978) Hospital-acquired mucormycosis (Rhizopus rhizopodiformis) of skin and subcutaneous tissue: epidemiology, mycology and treatment. N Engl J Med 299:1115-1118

4. Stave GM, Heimberger T, Kerkering TM (1989) Zygomycosis of the basal ganglia in intravenous drug users. Am J Med 86:115-117 